\title{
Fixed-Time Synchronization for Hybrid Coupled Dynamical Networks with Multilinks and Time-Varying Delays
}

\author{
Baolin Qiu, ${ }^{1,2}$ Lixiang Li, ${ }^{1,2}$ Haipeng Peng, ${ }^{1,2}$ and Yixian Yang ${ }^{1,2,3}$ \\ ${ }^{1}$ Information Security Center, State Key Laboratory of Networking and Switching Technology, \\ Beijing University of Posts and Telecommunications, Beijing 100876, China \\ ${ }^{2}$ National Engineering Laboratory for Disaster Backup and Recovery, Beijing University of Posts and Telecommunications, \\ Beijing 100876, China \\ ${ }^{3}$ State Key Laboratory of Public Big Data, Guizhou 550025, China \\ Correspondence should be addressed to Lixiang Li; li_lixiang2006@163.com
}

Received 28 September 2016; Revised 15 November 2016; Accepted 28 November 2016; Published 18 January 2017

Academic Editor: Sebastian Anita

Copyright (C) 2017 Baolin Qiu et al. This is an open access article distributed under the Creative Commons Attribution License, which permits unrestricted use, distribution, and reproduction in any medium, provided the original work is properly cited.

\begin{abstract}
This paper concerns the problem of fixed/finite-time synchronization of hybrid coupled dynamical networks. The considered dynamical networks with multilinks contain only one transmittal time-varying delay for each subnetwork, which makes us get hold of more interesting and practical points. Two kinds of delay-dependent feedback controllers with multilinks as well as appropriate Lyapunov functions are defined to achieve the goal of fixed-time synchronization and finite-time synchronization for the networks. Some novel and effective criteria of hybrid coupled networks are derived based on fixed-time and finite-time stability analysis. Finally, two numerical simulation examples are given to show the effectiveness of the results proposed in our paper.
\end{abstract}

\section{Introduction}

The phenomenon of synchronization exists everywhere in the realistic living world. People's applause begins with random rhythm, but it turns to the same rhythm at the end of a splendid drama. Every router of Internet periodically releases routing messages, respectively. However, the researchers have found that different router would eventually send routing messages in synchronous way, which led to the network traffic jam. Synchronization is common in every field and plays an important role. In fact, in physics, mathematics, and other fields, synchronous phenomenon of the coupled dynamical system has been studied widely in the past decade.

We are involved in a variety of complex dynamical networks, such as communication networks, Internet, transportation networks, and electricity networks. And synchronous phenomenon of dynamical networks is common. Sometimes synchronization is good for people's lives; sometimes synchronization may be harmful to people's lives and may even cause huge losses or serious consequences. Therefore, researches on synchronization of complex networks are of great value and significance. In a recent decade, researches on synchronization control of complex dynamical networks attract many researchers' attention [1, 2]. Meanwhile, lots of relevant achievements have been obtained. Researches on synchronization mainly concentrate on the following: complete synchronization $[3,4]$, projective synchronization [5-7], delay synchronization [8-10], finite-time synchronization [11-13], and so forth. Synchronization methods mainly contain the following: linear feedback method [14, 15], nonlinear feedback method [16], adaptive method [17], and so forth. According to the continuity of control law, synchronization techniques can be divided into continuous and noncontinuous synchronization control methods [1820]. Compared with continuous control law, noncontinuous control law can reduce some control cost.

Also, synchronization techniques can be divided into finite-time synchronization and infinite-time synchronization. Compared with the asymptotic stability and exponential stability, finite-time stability has relatively lower time complexity. Therefore, in the real world, obtaining synchronization in a setting time has more practical value. In secure 
communication, sending and receiving the information in a setting time can not only ensure the security of communication but also improve the efficiency and safety of communications [21]. However, achieving synchronization in finite time depends on the initial synchronization error value of the drive-response systems. The finite time for the unknown initial conditions of drive-response systems (the initial conditions of most of practical systems are hard to be achieved) is not fixed. Therefore, Polyakov defined the fixedtime stability theory. The time to obtain fixed synchronization is irrelevant to the initial synchronization error conditions of drive-response systems and only affected and controlled by the controller [22].

In this paper, we study the hybrid coupled dynamical networks containing coupling delay and internal delay that are indeed common in real world, which makes the research work more meaningful. In previous studies, coupling delay is defined as $D\left(x_{j}(t-\tau(t))-x_{i}(t-\tau(t))\right)$ [23-25]. In fact, as for the synchronization study of a pair of given driveresponse systems, the time delay influences the variables that are transmitted from one system to another system being studied. Therefore, considering the coupling term of time delay as $D\left(x_{j}(t-\tau(t))-x_{i}(t)\right)$ is more reasonable [26].

The concept of multilinks means that the edge number linking two nodes in complex networks is greater than one. Multilinked complex networks are ubiquitous in the real world, such as communication networks and transportation networks. Communication links of communication networks can be mail, telephone, QQ, letters, and so forth [27-29]. Compared with researches of single link networks [30-33], those of networks with multilinks are more practical and significant. There have been a lot of research achievements in this field so far [34-36]. However, to my best knowledge, few put their attention to the hybrid coupled networks with both one single time-varying delay coupling and multilinks. Few people study fixed-time synchronization and finite-time synchronization with the conditions of dynamical networks mentioned above. It is interesting to complete such study even to fill the gaps in this field.

Motivated by the foregoing analysis, this paper studies fixed-time synchronization and finite-time synchronization for hybrid coupled dynamical networks with multilinks and one single time-varying delay coupling. Furthermore, we propose new model of multilinked complex dynamical networks with one single time-varying delay coupling term and design two suitable feedback controllers to ensure the synchronization. With the constructed Lyapunov functions, several novel criteria are derived to achieve fixed-time/finitetime synchronization of the complex dynamical networks described in this paper. Finally, we give two numerical simulation examples to verify the effectiveness and correctness of our proposed theoretical results.

The rest of this paper is organized as follows. In Section 2, some preliminaries are introduced, such as notations, some assumptions, and lemmas. In Sections 3 and 4, the main results proposed in this paper are introduced. In Section 5, two numerical simulation examples are provided to verify the effectiveness and correctness of our obtained theoretical results. Finally, we draw the conclusion.

\section{Preliminaries}

Throughout this paper, we always have the functions $f(\cdot)$ and $g(\cdot)$ to satisfy the Lipschitz condition, which mean the following assumptions [37].

Assumption 1. For any $x, y \in R^{n}$, there exists a constant $p(p \geq 0)$ such that

$$
\|f(x)-f(y)\| \leq p\|x-y\| .
$$

Assumption 2. For any $x, y \in R^{n}$, there exists a constant $q(q \geq 0)$ such that

$$
\|g(x)-g(y)\| \leq q\|x-y\| .
$$

In order to prove the correctness of the results proposed in this paper, we need the following lemmas.

Lemma 3 (see [38]). For $x_{1}, x_{2}, x_{3}, \ldots, x_{N} \geq 0$ and $p>1,0<$ $q \leq 1$, two inequalities hold as follows:

$$
\begin{aligned}
& \sum_{i=1}^{N} x_{i}^{p} \geq N^{1-p}\left(\sum_{i=1}^{N} x_{i}\right)^{p}, \\
& \sum_{i=1}^{N} x_{i}^{q} \geq\left(\sum_{i=1}^{N} x_{i}\right)^{q} .
\end{aligned}
$$

Lemma 4 (see [39], (chain rule)). If $V(t): R^{n} \rightarrow R$ and $x(t)$ satisfy the following conditions,

(1) $V(t)$ is C-regular,

(2) $x(t)$ is absolutely continuous when it is on any compact subinterval of $[0,+\infty)$, then, the following holds:

$x(t)$ and $V(x(t)):[0,+\infty) \rightarrow R$ are differentiable for a.e.t $\in[0,+\infty)$;

$$
V(x(t))=v(t) \dot{x}(t), \quad \forall v(t) \in \partial V(x(t)),
$$

where $\partial V(x(t))$ is the Clarke generalized gradient of $V$ at the point $x(t)$.

Lemma 5 (see [40]). Let the function $V(t)$ be continuous and positive-definite, and $V(t)$ makes the differential inequalities hold as follows:

$$
\dot{V}(t) \leq-\kappa V^{\eta}(t) ; \quad \forall t \geq t_{0}, V\left(t_{0}\right) \geq 0,
$$

where $\eta \in(0,1)$ and $\kappa$ is positive constant. follows:

Then, for $\forall t_{0}, V(t)$ can always satisfy the inequality as

$$
V^{1-\eta}(t) \leq V^{1-\eta}\left(t_{0}\right)-\kappa(1-\eta)\left(t-t_{0}\right), \quad t_{0} \leq t \leq t_{1} .
$$

Also, $V(t) \equiv 0, \forall t \geq t_{1}$. The settling time $t_{1}$ is given as follows:

$$
t_{1}=t_{0}+\frac{V^{1-\eta}\left(t_{0}\right)}{\kappa(1-\eta)} .
$$

Lemma 6 (see [41]). Let the function $V(t)$ be continuous and positive-definite, and $V(t)$ makes the differential inequalities hold as follows:

$$
\dot{V}(t) \leq I V(t)-\kappa V^{\eta}(t), \quad \forall t \geq t_{0}, V^{1-\eta}\left(t_{0}\right) \leq \frac{\kappa}{I},
$$

where $\eta \in(0,1)$ and $\kappa$ and I are positive constant. 
Then, $V(t) \equiv 0, \forall t \geq t_{1}$, where the settling time $t_{1}$ is given as follows:

$$
t_{1}=t_{0}+\frac{\ln \left(1-(I / \kappa) V^{1-\eta}\left(t_{0}\right)\right)}{I(\eta-1)} .
$$

Lemma 7 (see [22]). Suppose that there exists a function $V$ : $R^{n N} \rightarrow R_{+} \cup 0$ which is continuous radically unbounded such that the following hold:

(1) If $x \neq(0, \ldots, 0)^{T}$, then $V(x)>0 ; V(x)=0 \Rightarrow x=$ $(0, \ldots, 0)^{T}$.

(2) Let $e(t)=\left(e_{1}^{T}(t), \ldots, e_{N}^{T}(t)\right)^{T}$ be any solution of error dynamical system. For some constants $a, b>0, p>$ $1,0<q<1, e(t)$ satisfies $\dot{V}(e(t)) \leq-a V^{p}(e(t))-$ $b V^{q}(e(t))$. Then, the following results hold:

$$
\begin{aligned}
V(e(t)) & \equiv 0, \quad \forall t \geq t_{0}, \\
T_{0} & =\frac{1}{a(p-1)}+\frac{1}{b(1-q)},
\end{aligned}
$$

where $T_{0}$ is the fixed settling time.

\section{Finite-Time Synchronization for Hybrid Coupled Networks with Multilinks}

In this section, we study finite-time synchronization for the multilinked complex dynamical networks with hybrid coupled terms and time-varying delays. By constructing Lyapunov function and designing appropriate controller, some useful criteria are derived to ensure that the multilinked complex dynamical networks proposed in this paper are synchronized.

3.1. Networks Model. The networks model is given as follows:

$$
\begin{aligned}
\dot{x}_{i}(t)= & f\left(x_{i}(t)\right)+g\left(x_{i}(t-\tau(t))\right) \\
& +\sum_{j=1, j \neq i}^{N} G_{i j}^{0} D\left(x_{j}(t)-x_{i}(t)\right) \\
& +\sum_{j=1, j \neq i}^{N} G_{i j}^{1} D_{\tau_{1}}\left(x_{j}\left(t-\tau_{1}(t)\right)-x_{i}(t)\right)+\cdots \\
& +\sum_{j=1, j \neq i}^{N} G_{i j}^{m} D_{\tau_{m}}\left(x_{j}\left(t-\tau_{m}(t)\right)-x_{i}(t)\right),
\end{aligned}
$$

where $i=1,2, \ldots, N$ is the number of nodes and $x_{i}(t)=$ $\left(x_{i 1}(t), x_{i 2}(t), \ldots, x_{i n}(t)\right)^{T} \in R^{n}$ is the state vector of the $i$ th node of the network; vector functions $f, g: R^{n} \rightarrow R^{n}$ are continuous differentiable; $\tau(t)$ is internal delay; $\tau(t) \leq$ $\tau, \tau$ is a positive constant; $\tau_{k}(t)(k=1,2,3, \ldots, m)$ denotes the delay of the $k$ th subnetwork correspondingly, and $0 \leq$ $\tau_{k}(t) \leq \tau ; D=\left(D_{i j}\right)_{n \times n}$ denotes the inner coupling matrix of the 0th subnetwork between node $i$ and node $j$ at the moment $t$; correspondingly, $D_{\tau_{k}}(k=1,2,3, \ldots, m)$ denotes the inner coupling matrix of the $k$ th subnetwork between node $i$ and node $j$ at the moment $t-\tau_{k}(t) ; G^{k}=\left(G_{i j}^{k}\right)_{n \times n}$ $(k=0,1,2, \ldots, m)$ denotes the configuration matrix of the $k$ th subnetwork and satisfies the conditions as follows:

$$
G_{i i}^{k}=-\sum_{j=1, j \neq i}^{N} G_{i j}^{k}, \quad G_{i j}^{k}=G_{j i}^{k} \geq 0, i \neq j,
$$

where $k=0,1,2, \ldots, m ; G_{i j}^{k}>0$ means there exists a connection between nodes $i$ and $j$; otherwise, $G_{i j}^{k}=0$.

Note 1. We propose a new multilinked complex dynamical networks model with hybrid coupled terms and time-varying delays. Bringing in inner coupling matrices $D \& D_{\tau_{k}}$, our study of the finite/fixed-time synchronization for the hybrid coupled dynamical networks with both one single timevarying delay coupling and multilinks is more practical for the real world. To the best of our knowledge, this study is the first exploration in such a field.

Consider system (11) as the drive system; correspondingly, the response system is described as follows:

$$
\begin{aligned}
\dot{y}_{i}(t)= & f\left(y_{i}(t)\right)+g\left(y_{i}(t-\tau(t))\right) \\
& +\sum_{j=1, j \neq i}^{N} G_{i j}^{0} D\left(y_{j}(t)-y_{i}(t)\right) \\
& +\sum_{j=1, j \neq i}^{N} G_{i j}^{1} D_{\tau_{1}}\left(y_{j}\left(t-\tau_{1}(t)\right)-y_{i}(t)\right)+\cdots \\
& +\sum_{j=1, j \neq i}^{N} G_{i j}^{m} D_{\tau_{m}}\left(y_{j}\left(t-\tau_{m}(t)\right)-y_{i}(t)\right) \\
& +u_{i}(t),
\end{aligned}
$$

where $y_{i}(t)=\left(y_{i 1}(t), y_{i 2}(t), \ldots, y_{i n}(t)\right)^{T} \in R^{n}$ is the response state vector of the $i$ th node for the response system; $u_{i}(t)(i=1,2, \ldots, N)$ is the designed controller to achieve synchronization.

Assume that $C\left([-\tau, 0], R^{n}\right)$ is a Banach space which consists of continuous functions mapping the interval $[-\tau, 0]$ into $R^{n}$ with the norm $\|\phi\|=\sup _{-\tau \leq \theta \leq 0}\|\phi(\theta)\|$. For the drive system (11) and the response system (13), their initial conditions are, respectively, given by $x_{i}(t)=\phi_{i}(t) \in C\left([-\tau, 0], R^{n}\right)$ and $y_{i}(t)=\varphi_{i}(t) \in C\left([-\tau, 0], R^{n}\right)$. Assume that in each of model equation (11) and model equation (13) a unique solution exists in regard to the initial conditions mentioned.

In order to use networks model equations conveniently, we rewrite equation (11) and equation (13) with the comprehensive consideration of condition (12). The results are given as follows:

$$
\begin{aligned}
\dot{x}_{i}(t)= & f\left(x_{i}(t)\right)+g\left(x_{i}(t-\tau(t))\right)+\sum_{j=1}^{N} G_{i j}^{0} D x_{j}(t) \\
& +\sum_{j=1}^{N} G_{i j}^{1} D_{\tau_{1}} x_{j}\left(t-\tau_{1}(t)\right)+\cdots
\end{aligned}
$$




$$
\begin{aligned}
& +\sum_{j=1}^{N} G_{i j}^{m} D_{\tau_{m}} x_{j}\left(t-\tau_{m}(t)\right) \\
& -G_{i i}^{1} D_{\tau_{1}}\left(x_{i}\left(t-\tau_{1}(t)\right)-x_{i}(t)\right)-\cdots \\
& -G_{i i}^{m} D_{\tau_{m}}\left(x_{i}\left(t-\tau_{m}(t)\right)-x_{i}(t)\right), \\
\dot{y}_{i}(t)= & f\left(y_{i}(t)\right)+g\left(y_{i}(t-\tau(t))\right)+\sum_{j=1}^{N} G_{i j}^{0} D y_{j}(t) \\
& +\sum_{j=1}^{N} G_{i j}^{1} D_{\tau_{1}} y_{j}\left(t-\tau_{1}(t)\right)+\cdots \\
& +\sum_{j=1}^{N} G_{i j}^{m} D_{\tau_{m}} y_{j}\left(t-\tau_{m}(t)\right) \\
& -G_{i i}^{1} D_{\tau_{1}}\left(y_{i}\left(t-\tau_{1}(t)\right)-y_{i}(t)\right)-\cdots \\
& -G_{i i}^{m} D_{\tau_{m}}\left(y_{i}\left(t-\tau_{m}(t)\right)-y_{i}(t)\right)+u_{i}(t) .
\end{aligned}
$$

Let the synchronization errors $e_{i}(t)=y_{i}(t)-x_{i}(t), i=$ $1,2, \ldots, N$. Then we have

$$
\begin{aligned}
\dot{e}_{i}(t)= & \dot{y}_{i}(t)-\dot{x}_{i}(t) \\
= & f\left(y_{i}(t)\right)-f\left(x_{i}(t)\right)+g\left(y_{i}(t-\tau(t))\right) \\
& -g\left(x_{i}(t-\tau(t))\right)+\sum_{j=1}^{N} G_{i j}^{0} D e_{j}(t) \\
& +\sum_{j=1}^{N} G_{i j}^{1} D_{\tau_{1}} e_{j}\left(t-\tau_{1}(t)\right)+\cdots \\
& +\sum_{j=1}^{N} G_{i j}^{m} D_{\tau_{m}} e_{j}\left(t-\tau_{m}(t)\right) \\
& -G_{i i}^{1} D_{\tau_{1}}\left(e_{i}\left(t-\tau_{1}(t)\right)-e_{i}(t)\right)-\cdots \\
& -G_{i i}^{m} D_{\tau_{m}}\left(e_{i}\left(t-\tau_{m}(t)\right)-e_{i}(t)\right)+u_{i}(t) .
\end{aligned}
$$

3.2. Finite-Time Synchronization between the Drive System and the Response System. Combining with the given lemmas, assumptions, and conditions, we study finite-time stability of the origin of the given error dynamical system, which denotes the finite-time synchronization for the multilinked complex dynamical networks (11) and (13).

Definition 8 (the finite-time stability). Through controlling of the designed controller, there always exists a positive constant $t_{1}$, and the value of $t_{1}$ depends on the value of the initial state vector $e_{i}(t)=\Phi_{i}(t) \in C\left([-\tau, 0], R^{n}\right)$ for $t \in[-\tau, 0]$. If the following expressions hold

$$
\begin{aligned}
\lim _{t \rightarrow t_{1}} e_{i}(t) & =(0,0, \ldots, 0)^{T}, \\
& e_{i}(t)=(0,0, \ldots, 0)^{T}, t>t_{1}, i=1,2, \ldots, N,
\end{aligned}
$$

then, the error dynamical system (15) is said to be finite-time stable, and this state of the error dynamical system is named as the finite-time stability. And $t_{1}$ is called the settling time.

In order to obtain the target of finite-time synchronization for systems (11) and (13), we design the following controller as the control law of complex dynamical networks (13). The control law is given as follows:

$$
\begin{aligned}
& u_{i}(t)=-\sqrt{n} \cdot \operatorname{sign}\left(e_{i}(t)\right)\left[p_{i}\left\|e_{i}(t)\right\|_{1}\right. \\
& +q_{i}\left\|e_{i}(t-\tau(t))\right\|_{1}+\sum_{k=1}^{m} \xi_{i}^{k}\left\|e_{i}\left(t-\tau_{k}(t)\right)\right\|_{1} \\
& \left.+\frac{k_{0}}{n^{(\eta / 2)}}\left\|e_{i}(t)\right\|_{1}^{\eta}\right]
\end{aligned}
$$

where $k_{0}>0,0<\eta<1$, and $k=1,2, \ldots, m$; the parameters of $p_{i}, q_{i}, \xi_{i}^{k}$ will be determined as follows.

The following theorem can be derived for the driveresponse systems of models (11) and (13) with the given controller (17).

Theorem 9. Let Assumptions 1 and 2 hold. If there exist the positive parameters $p_{i}, q_{i}, \xi_{i}^{k}$ such that the following inequalities hold

$$
\begin{aligned}
& p_{i} \geq \alpha+2\left|G_{i i}^{0}\right|\|D\|+\sum_{k^{\prime}=1}^{m}\left|G_{i i}^{k^{\prime}}\right|\left\|D_{\tau_{k^{\prime}}}\right\|, \\
& q_{i} \geq \beta, \\
& \xi_{i}^{k} \geq 3\left|G_{i i}^{k}\right|\left\|D_{\tau_{k}} \mid\right\|, \quad k=1,2, \ldots, m,
\end{aligned}
$$

then, the drive system (11) and response system (13) can realize the synchronization in finite time. And the settling time $t_{1}$ can be determined as $t_{1}=t_{0}+V^{1-\eta}\left(t_{0}\right) / k_{0}(1-\eta)$, where $V\left(t_{0}\right)=$ $\sum_{i=1}^{N}\left(\left\|e_{i}\left(t_{0}\right)\right\|_{1} / \sqrt{n}\right)$.

Proof. In this paper, the Lyapunov function is constructed as follows:

$$
V(t, e(t))=\sum_{i=1}^{N} \frac{\left\|e_{i}(t)\right\|_{1}}{\sqrt{n}}=\sum_{i=1}^{N} \frac{\operatorname{sign}^{T}\left(e_{i}(t)\right) e_{i}(t)}{\sqrt{n}} .
$$

Applying Lemma 4 and the error system to the process of the proof, we calculate the derivative of $V(t)$ step by step as follows:

$$
\begin{aligned}
\dot{V}(t) & =\sum_{i=1}^{N} \frac{\operatorname{sign}^{T}\left(e_{i}(t)\right) \dot{e}_{i}(t)}{\sqrt{n}} \\
& =\sum_{i=1}^{N} \frac{\operatorname{sign}^{T}\left(e_{i}(t)\right)}{\sqrt{n}}\left[f\left(y_{i}(t)\right)-f\left(x_{i}(t)\right)\right.
\end{aligned}
$$




$$
\begin{aligned}
& +g\left(y_{i}(t-\tau(t))\right)-g\left(x_{i}(t-\tau(t))\right) \\
& +\sum_{j=1}^{N} G_{i j}^{0} D e_{j}(t)+\sum_{j=1}^{N} G_{i j}^{1} D_{\tau_{1}} e_{j}\left(t-\tau_{1}(t)\right)+\cdots \\
& +\sum_{j=1}^{N} G_{i j}^{m} D_{\tau_{m}} e_{j}\left(t-\tau_{m}(t)\right) \\
& -G_{i i}^{1} D_{\tau_{1}}\left(e_{i}\left(t-\tau_{1}(t)\right)-e_{i}(t)\right)-\cdots \\
& \left.-G_{i i}^{m} D_{\tau_{m}}\left(e_{i}\left(t-\tau_{m}(t)\right)-e_{i}(t)\right)+u_{i}(t)\right] \\
& \leq \sum_{i=1}^{N} \frac{1}{\sqrt{n}}\left[\left\|\operatorname{sign}^{T}\left(e_{i}(t)\right)\right\|\left\|f\left(y_{i}(t)\right)-f\left(x_{i}(t)\right)\right\|\right. \\
& +\left\|\operatorname{sign}^{T}\left(e_{i}(t)\right)\right\| \\
& \cdot\left\|g\left(y_{i}(t-\tau(t))\right)-g\left(x_{i}(t-\tau(t))\right)\right\| \\
& +\left\|\operatorname{sign}^{T}\left(e_{i}(t)\right)\right\| \sum_{j=1}^{N}\left|G_{i j}^{0}\right|\|D\|\left\|e_{j}(t)\right\| \\
& +\left\|\operatorname{sign}^{T}\left(e_{i}(t)\right)\right\| \sum_{j=1}^{N}\left|G_{i j}^{1}\right|\left\|D_{\tau_{1}}\right\|\left\|e_{j}\left(t-\tau_{1}(t)\right)\right\| \\
& +\cdots+\left\|\operatorname{sign}^{T}\left(e_{i}(t)\right)\right\| \\
& \cdot \sum_{j=1}^{N}\left|G_{i j}^{m}\right|\left\|D_{\tau_{m}}\right\|\left\|e_{j}\left(t-\tau_{m}(t)\right)\right\|+\left\|\operatorname{sign}^{T}\left(e_{i}(t)\right)\right\| \\
& \cdot\left|G_{i i}^{1}\right|\left\|D_{\tau_{1}}\right\|\left(\left\|e_{i}\left(t-\tau_{1}(t)\right)\right\|+\left\|e_{i}(t)\right\|\right)+\cdots \\
& +\left\|\operatorname{sign}^{T}\left(e_{i}(t)\right)\right\|\left|G_{i i}^{m}\right|\left\|D_{\tau_{m}}\right\| \\
& \cdot\left(\left\|e_{i}\left(t-\tau_{m}(t)\right)\right\|+\left\|e_{i}(t)\right\|\right) \\
& \left.+\operatorname{sign}^{T}\left(e_{i}(t)\right) u_{i}(t)\right] \leq \sum_{i=1}^{N} \alpha\left\|e_{i}(t)\right\| \\
& +\sum_{i=1}^{N} \beta\left\|e_{i}(t-\tau(t))\right\|+\sum_{i=1}^{N} \sum_{j=1}^{N}\left|G_{i j}^{0}\right|\|D\|\left\|e_{j}(t)\right\| \\
& \cdot\left\|D_{\tau_{1}}\right\|\left(\left\|e_{i}\left(t-\tau_{1}(t)\right)\right\|+\left\|e_{i}(t)\right\|\right)+\cdots+\sum_{i=1}^{N}\left|G_{i i}^{m}\right| \\
& \cdot\left\|D_{\tau_{m}}\right\|\left(\left\|e_{i}\left(t-\tau_{m}(t)\right)\right\|+\left\|e_{i}(t)\right\|\right) \\
& +\sum_{i=1}^{N} \frac{\operatorname{sign}^{T}\left(e_{i}(t)\right)}{\sqrt{n}} u_{i}(t)=\sum_{i=1}^{N} \alpha\left\|e_{i}(t)\right\| \\
& +\sum_{i=1}^{N} \beta\left\|e_{i}(t-\tau(t))\right\|+\sum_{i=1}^{N} 2\left|G_{i i}^{0}\right|\|D\|\left\|e_{i}(t)\right\| \\
& +\sum_{i=1}^{N} 2\left|G_{i i}^{1}\right|\left\|D_{\tau_{1}}\right\|\left\|e_{i}\left(t-\tau_{1}(t)\right)\right\|+\cdots+\sum_{i=1}^{N} 2\left|G_{i i}^{m}\right| \\
& \cdot\left\|D_{\tau_{m}}\right\|\left\|e_{i}\left(t-\tau_{m}(t)\right)\right\|+\sum_{i=1}^{N}\left|G_{i i}^{1}\right|\left\|D_{\tau_{1}}\right\| \\
& \cdot\left\|e_{i}\left(t-\tau_{1}(t)\right)\right\|+\sum_{i=1}^{N}\left|G_{i i}^{1}\right|\left\|D_{\tau_{1}}\right\|\left\|e_{i}(t)\right\|+\cdots \\
& +\sum_{i=1}^{N}\left|G_{i i}^{m}\right|\left\|D_{\tau_{m}}\right\|\left\|e_{i}\left(t-\tau_{m}(t)\right)\right\|+\sum_{i=1}^{N}\left|G_{i i}^{m}\right|\left\|D_{\tau_{m}}\right\| \\
& \cdot\left\|e_{i}(t)\right\|+\sum_{i=1}^{N} \frac{\operatorname{sign}^{T}\left(e_{i}(t)\right)}{\sqrt{n}} u_{i}(t)=\sum_{i=1}^{N}\left(\alpha+2\left|G_{i i}^{0}\right|\right. \\
& \left.\cdot\|D\|+\sum_{k^{\prime}=1}^{m}\left|G_{i i}^{k^{\prime}}\right|\left\|D_{\tau_{k^{\prime}}}\right\|\right)\left\|e_{i}(t)\right\| \\
& +\sum_{i=1}^{N} \beta\left\|e_{i}(t-\tau(t))\right\|+\sum_{i=1}^{N} \sum_{k=1}^{m} 3\left|G_{i i}^{k}\right|\left\|D_{\tau_{k}}\right\| \\
& \cdot\left\|e_{i}\left(t-\tau_{k}(t)\right)\right\|+\sum_{i=1}^{N} \frac{\operatorname{sign}^{T}\left(e_{i}(t)\right)}{\sqrt{n}} u_{i}(t) \leq \sum_{i=1}^{N}(\alpha \\
& \left.+2\left|G_{i i}^{0}\right|\|D\|+\sum_{k^{\prime}=1}^{m}\left|G_{i i}^{k^{\prime}}\right|\left\|D_{\tau_{k^{\prime}}}\right\|\right)\left\|e_{i}(t)\right\|_{1} \\
& +\sum_{i=1}^{N} \beta\left\|e_{i}(t-\tau(t))\right\|_{1}+\sum_{i=1}^{N} \sum_{k=1}^{m} 3\left|G_{i i}^{k}\right|\left\|D_{\tau_{k}}\right\| \\
& \cdot\left\|e_{i}\left(t-\tau_{k}(t)\right)\right\|_{1}+\sum_{i=1}^{N} \frac{\operatorname{sign}^{T}\left(e_{i}(t)\right)}{\sqrt{n}} u_{i}(t) .
\end{aligned}
$$$$
+\sum_{i=1}^{N} \sum_{j=1}^{N}\left|G_{i j}^{1}\right|\left\|D_{\tau_{1}}\right\|\left\|e_{j}\left(t-\tau_{1}(t)\right)\right\|+\cdots
$$$$
+\sum_{i=1}^{N} \sum_{j=1}^{N}\left|G_{i j}^{m}\right|\left\|D_{\tau_{m}}\right\|\left\|e_{j}\left(t-\tau_{m}(t)\right)\right\|+\sum_{i=1}^{N}\left|G_{i i}^{1}\right|
$$

The calculating result (20) contains many details. Firstly, in order to calculate conveniently later, we deal with the function $\sum_{i=1}^{N}\left(\left(\operatorname{sign}^{T}\left(e_{i}(t)\right) / \sqrt{n}\right) u_{i}(t)\right)$ in $(20)$. Then, we substitute the partial processing result into the previous result (20). We get 


$$
\begin{aligned}
& \sum_{i=1}^{N} \frac{\operatorname{sign}^{T}\left(e_{i}(t)\right)}{\sqrt{n}} u_{i}(t) \\
& \quad=\sum_{i=1}^{N} \frac{\operatorname{sign}^{T}\left(e_{i}(t)\right)}{\sqrt{n}}\left\{-\sqrt{n} \cdot \operatorname{sign}\left(e_{i}(t)\right)\left[p_{i}\left\|e_{i}(t)\right\|_{1}+q_{i}\left\|e_{i}(t-\tau(t))\right\|_{1}+\sum_{k=1}^{m} \xi_{i}^{k}\left\|e_{i}\left(t-\tau_{k}(t)\right)\right\|_{1}+\frac{k_{0}}{n^{(\eta / 2)}}\left\|e_{i}(t)\right\|_{1}^{\eta}\right]\right\} \\
& \quad \leq-\left[\sum_{i=1}^{N} p_{i}\left\|e_{i}(t)\right\|_{1}+\sum_{i=1}^{N} q_{i}\left\|e_{i}(t-\tau(t))\right\|_{1}+\sum_{i=1}^{N} \sum_{k=1}^{m} \xi_{i}^{k}\left\|e_{i}\left(t-\tau_{k}(t)\right)\right\|_{1}+\sum_{i=1}^{N} \frac{k_{0}}{n^{(\eta / 2)}}\left\|e_{i}(t)\right\|_{1}^{\eta}\right] .
\end{aligned}
$$

Secondly, we substitute result (21) into result (20). We get the following result:

$$
\begin{gathered}
\dot{V}(t) \leq \sum_{i=1}^{N}\left(\alpha+2\left|G_{i i}^{0}\right|\|D\|+\sum_{k^{\prime}=1}^{m}\left|G_{i i}^{k^{\prime}}\right|\left\|D_{\tau_{k^{\prime}}}\right\|-p_{i}\right) \\
\cdot\left\|e_{i}(t)\right\|_{1}+\sum_{i=1}^{N}\left(\beta-q_{i}\right)\left\|e_{i}(t-\tau(t))\right\|_{1} \\
+\sum_{i=1}^{N} \sum_{k=1}^{m}\left(3\left|G_{i i}^{k}\right|\left\|D_{\tau_{k}}\right\|-\xi_{i}^{k}\right)\left\|e_{i}\left(t-\tau_{k}(t)\right)\right\|_{1} \\
-\sum_{i=1}^{N} \frac{k_{0}}{n^{(\eta / 2)}}\left\|e_{i}(t)\right\|_{1}^{\eta} \leq-\sum_{i=1}^{N} \frac{k_{0}}{n^{(\eta / 2)}}\left\|e_{i}(t)\right\|_{1}^{\eta} \\
\leq-k_{0}\left(\sum_{i=1}^{N} \frac{\left\|e_{i}(t)\right\|_{1}}{\sqrt{n}}\right)^{\eta}=-k_{0} V^{\eta}(t) .
\end{gathered}
$$

According to Lemma 5, the error dynamical system (15) can be stable in finite time. And the settling time is determined by $t_{1}=t_{0}+V^{1-\eta}\left(t_{0}\right) / k_{0}(1-\eta)$.

This completes the proof.

Remark 10. Lemma 5 plays the role of an important theoretical basis for the previous proof, which supports the analysis of finite-time synchronization for hybrid coupled complex networks with multilinks and internal/coupling delay terms with single time-varying delays. Lemma 5 can be traced from [40].

According to Lemma 6, the following corollary can be obtained.

Corollary 11. Let both Assumptions 1 and 2 hold. The designed controller is described as follows:

$$
\begin{aligned}
& u_{i}(t)=-\sqrt{n} \cdot \operatorname{sign}\left(e_{i}(t)\right)\left(p_{i}\left\|e_{i}(t)\right\|_{1}\right. \\
& +q_{i}\left\|e_{i}(t-\tau(t))\right\|_{1}+\sum_{k=1}^{m} \xi_{i}^{k}\left\|e_{i}\left(t-\tau_{k}(t)\right)\right\|_{1} \\
& \left.+\frac{k_{0}}{n^{(\eta / 2)}}\left\|e_{i}(t)\right\|_{1}^{\eta}\right)
\end{aligned}
$$

where $k_{0}>0$ and $0<\eta<1$.
And let the parameters $p_{i}, q_{i}, \xi_{i}^{k}$ satisfy the following inequalities:

$$
\begin{aligned}
& p_{i}<\alpha+2\left|G_{i i}^{0}\right|\|D\|+\sum_{k^{\prime}=1}^{m}\left|G_{i i}^{k^{\prime}}\right|\left\|D_{\tau_{k^{\prime}}}\right\|, \\
& q_{i} \geq \beta, \\
& \xi_{i}^{k} \geq 3\left|G_{i i}^{k}\right|\left\|D_{\tau_{k}} \mid\right\|, \quad k=1,2, \ldots, m,
\end{aligned}
$$

where $p_{i}>0, q_{i}>0$, and $\xi_{i}^{k}>0$. Besides, $V^{1-\eta} \leq k_{0} /[\sqrt{n}(\alpha+$ $\left.\left.2\left|G_{i i}^{0}\right|\|D\|+\sum_{k^{\prime}=1}^{m}\left|G_{i i}^{k^{\prime}}\right|\left\|D_{\tau_{k^{\prime}}}\right\|-p_{i}\right)\right]$.

Then, we can obtain the conclusion that the complex system (11) and complex system (13) will realize synchronization in finite time.

Remark 12. According to Theorem 9 and Corollary 11, some sufficient finite-time synchronization criteria of the multilinked hybrid coupled networks are derived for the driveresponse systems of models (11) and (13) with the designed controller in this paper. The parameters $p_{i}>0, q_{i}>0$, and $\xi_{i}^{k}>0(k=1,2, \ldots, m)$ are critical to the finite-time synchronization of networks (11) and (13). Compared with the previous researches, the models of complex dynamical networks proposed in our paper are more general.

\subsection{Finite-Time Synchronization between Hybrid Coupled} Dynamical Network and an Isolated Node. Suppose that we need multilinked hybrid coupled complex networks to synchronize to an isolated node in finite time. Then we will design the following complex networks model (25) as follows:

$$
\begin{aligned}
\dot{x}_{i}(t)= & f\left(x_{i}(t)\right)+g\left(x_{i}(t-\tau(t))\right)+\sum_{j=1}^{N} G_{i j}^{0} D x_{j}(t) \\
& +\sum_{j=1}^{N} G_{i j}^{1} D_{\tau_{1}} x_{j}\left(t-\tau_{1}(t)\right)+\cdots \\
& +\sum_{j=1}^{N} G_{i j}^{m} D_{\tau_{m}} x_{j}\left(t-\tau_{m}(t)\right) \\
& -G_{i i}^{1} D_{\tau_{1}}\left(x_{i}\left(t-\tau_{1}(t)\right)-x_{i}(t)\right)-\cdots \\
& -G_{i i}^{m} D_{\tau_{m}}\left(x_{i}\left(t-\tau_{m}(t)\right)-x_{i}(t)\right)+u_{i}(t),
\end{aligned}
$$

where $i=1,2, \ldots, N$. 
If the finite-time synchronization is obtained, that is, $x_{1}(t)=x_{2}(t)=\cdots=x_{N}(t)$, the synchronized state model can be determined as follows:

$$
\begin{aligned}
\dot{s}(t)= & f(s(t))+g(s(t-\tau(t))) \\
& -G_{i i}^{1} D_{\tau_{1}}\left(s\left(t-\tau_{1}(t)\right)-s(t)\right)-\cdots \\
& -G_{i i}^{m} D_{\tau_{m}}\left(s\left(t-\tau_{m}(t)\right)-s(t)\right),
\end{aligned}
$$

where $i=1,2, \ldots, N$. Apparently, all of $x_{1}(t), x_{2}(t), \ldots, x_{N}(t)$ must not only satisfy $x_{1}(t)=x_{2}(t)=\ldots=x_{N}(t)$ but also be invariant for different $i$ while the finite-time synchronization is realized. But equation (26) does not meet the requirement. Therefore, we need the following assumption to be satisfied in order to complete the proof of this proposed supposition.

Assumption 13. For the configuration matrices $G^{k}(k=$ $1,2, \ldots, m)$ of multilinked hybrid coupled complex network, $G_{i i}^{k}=G_{j j}^{k}=-a_{k}$, where $k=0,1,2, \ldots, m ; i=1,2, \ldots, N$; $j=1,2, \ldots, N$; and all of $a_{1}, a_{2}, \ldots, a_{m}$ are positive constants.

Use $\omega_{i}(t)$ to denote the synchronization errors, where $\omega_{i}(t)=x_{i}(t)-s(t), i=1,2, \ldots, N$. Combining with the given condition (12), $\omega_{i}(t)$ can be described as follows:

$$
\begin{aligned}
\dot{\omega}_{i}(t)= & \dot{x}_{i}(t)-\dot{s}(t) \\
= & f\left(x_{i}(t)\right)-f(s(t))+g\left(x_{i}(t-\tau(t))\right) \\
& -g(s(t-\tau(t)))+\sum_{j=1}^{N} G_{i j}^{0} D \omega_{j}(t) \\
& +\sum_{j=1}^{N} G_{i j}^{1} D_{\tau_{1}} \omega_{j}\left(t-\tau_{1}(t)\right)+\cdots \\
& +\sum_{j=1}^{N} G_{i j}^{m} D_{\tau_{m}} \omega_{j}\left(t-\tau_{m}(t)\right) \\
& -G_{i i}^{1} D_{\tau_{1}}\left(\omega_{i}\left(t-\tau_{1}(t)\right)-\omega_{i}(t)\right)-\cdots \\
& -G_{i i}^{m} D_{\tau_{m}}\left(\omega_{i}\left(t-\tau_{m}(t)\right)-\omega_{i}(t)\right)+u_{i}(t) .
\end{aligned}
$$

The process of the proof for system (25) and isolated node (26) to obtain finite-time synchronization is the same as that for Theorem 9. Hence, we omit it.

Then, the following corollary can easily be obtained.

Corollary 14. Let all of Assumptions 1, 2, and 13 hold. Suppose that there exist constants $p_{i}, q_{i}, \xi_{i}^{k}$ satisfying the following inequalities:

$$
\begin{aligned}
& p_{i} \geq \alpha+2 a_{0}\|D\|+\sum_{k^{\prime}=1}^{m} a_{k^{\prime}}\left\|D_{\tau_{k^{\prime}}}\right\|, \\
& q_{i} \geq \beta, \\
& \xi_{i}^{k} \geq 3 a_{k}\left\|D_{\tau_{k}}\right\|, \quad k=1,2, \ldots, m,
\end{aligned}
$$

where $p_{i}>0, q_{i}>0$, and $\xi_{i}^{k}>0$.
The controller designed to obtain the finite-time synchronization is described as follows:

$$
\begin{aligned}
& u_{i}(t)=-\sqrt{n} \cdot \operatorname{sign}\left(\omega_{i}(t)\right)\left(p_{i}\left\|\omega_{i}(t)\right\|_{1}\right. \\
& +q_{i}\left\|\omega_{i}(t-\tau(t))\right\|_{1}+\sum_{k=1}^{m} \xi_{i}^{k}\left\|\omega_{i}\left(t-\tau_{k}(t)\right)\right\|_{1} \\
& \left.+\frac{k_{0}}{n^{(\eta / 2)}}\left\|\omega_{i}(t)\right\|_{1}^{\eta}\right),
\end{aligned}
$$

where $k_{0}>0$ and $0<\eta<1$.

Then, the multilinked hybrid coupled complex networks (25) with control law (29) can realize the finite-time synchronization.

\section{Fixed-Time Synchronization for Hybrid Coupled Networks with Multilinks}

In this section, combining with the given lemmas, assumptions, conditions, and so on, we study the fixed-time stability of the origin for the given error dynamical system (15), which denotes the fixed-time synchronization for the multilinked complex dynamical networks (11) and (13).

So, we need to define what the fixed-time stability is, and its definition is described as follows.

Definition 15 (the fixed-time stability). Through controlling of the designed controller, there always exists a positive and fixed constant $T_{0}$, and the value of $T_{0}$ is irrelevant to the initial conditions. If the following expressions hold

$$
\begin{aligned}
& \lim _{t \rightarrow T_{0}} e_{i}(t)=(0,0, \ldots, 0)^{T}, \\
& \quad e_{i}(t) \equiv(0,0, \ldots, 0)^{T}, t>T_{0}, i=1,2, \ldots, N,
\end{aligned}
$$

then, the error dynamical system (15) is said to be fixed-time stable; the state of the error dynamical system is called the fixed-time stability. And $T_{0}$ is named as the fixed settling time.

Note 2. Through analyzing finite-time stability and fixedtime stability, we can easily find that finite-time synchronization depends on the initial conditions of the complex networks; on the contrary, fixed-time synchronization is irrelevant to the initial conditions of the complex networks. Therefore, it is meaningful to study fixed-time synchronization, especially when the initial conditions are hard to be achieved.

The control law designed to obtain the fixed-time synchronization is given as follows:

$$
\begin{aligned}
& u_{i}(t)=-\sqrt{n} \cdot \operatorname{sign}\left(e_{i}(t)\right)\left(p_{i}\left\|e_{i}(t)\right\|_{1}\right. \\
& +q_{i}\left\|e_{i}(t-\tau(t))\right\|_{1}+\sum_{k=1}^{m} \xi_{i}^{k}\left\|e_{i}\left(t-\tau_{k}(t)\right)\right\|_{1} \\
& \left.+\frac{a\left\|e_{i}(t)\right\|_{1}^{p}}{n^{(p / 2)}}+\frac{b\left\|e_{i}(t)\right\|_{1}^{q}}{n^{(q / 2)}}\right),
\end{aligned}
$$


where $a, b>0, p>1,0<q<1, k=1,2, \ldots, m$, and the parameters $p_{i}, q_{i}$, and $\xi_{i}^{k}$ will be determined later on.

Theorem 16. Let Assumptions 1 and 2 hold. If there exist the positive parameters $p_{i}, q_{i}, \xi_{i}^{k}(k=1,2, \ldots, m)$ such that the following inequalities hold

$$
\begin{aligned}
& p_{i} \geq \alpha+2\left|G_{i i}^{0}\right|\|D\|+\sum_{k^{\prime}=1}^{m}\left|G_{i i}^{k^{\prime}}\right|\left\|D_{\tau_{k^{\prime}}}\right\|, \\
& q_{i} \geq \beta, \\
& \xi_{i}^{k} \geq 3\left|G_{i i}^{k}\right|\left\|D_{\tau_{k}}\right\|, \quad k=1,2, \ldots, m,
\end{aligned}
$$

then, according to Lemma 7, the drive system (11) and response system (13) can realize synchronization in fixed-time $T_{0}$. And the fixed settling time $T_{0}$ can be determined as $T_{0}=$ $1 / a N^{1-p}(p-1)+1 / b(1-q)$.

The following is the process of the proof.

Proof. The designed Lyapunov function is given as follows:

$$
\begin{aligned}
V(t, e(t)) & =\sum_{i=1}^{N} \frac{\left\|e_{i}(t)\right\|_{1}}{\sqrt{n}} \\
& =\sum_{i=1}^{N} \frac{\operatorname{sign}^{T}\left(e_{i}(t)\right) e_{i}(t)}{\sqrt{n}},
\end{aligned}
$$

where $e(t)=\left(e_{1}^{T}(t), e_{2}^{T}(t), \ldots, e_{n}^{T}(t)\right)^{T}$.

$$
\begin{aligned}
& \sum_{i=1}^{N} \frac{\operatorname{sign}^{T}\left(e_{i}(t)\right)}{\sqrt{n}} u_{i}(t)=\sum_{i=1}^{N} \frac{\operatorname{sign}^{T}\left(e_{i}(t)\right)}{\sqrt{n}}[-\sqrt{n} \\
& \left.\quad \cdot \operatorname{sign}\left(e_{i}(t)\right)\left(p_{i}\left\|e_{i}(t)\right\|_{1}+q_{i}\left\|e_{i}(t-\tau(t))\right\|_{1}+\sum_{k=1}^{m} \xi_{i}^{k}\left\|e_{i}\left(t-\tau_{k}(t)\right)\right\|_{1}+\frac{a\left\|e_{i}(t)\right\|_{1}^{p}}{n^{(p / 2)}}+\frac{b\left\|e_{i}(t)\right\|_{1}^{q}}{n^{(q / 2)}}\right)\right] \\
& \quad \leq-\left(\sum_{i=1}^{N} p_{i}\left\|e_{i}(t)\right\|_{1}+\sum_{i=1}^{N} q_{i}\left\|e_{i}(t-\tau(t))\right\|_{1}+\sum_{i=1}^{N} \sum_{k=1}^{m} \xi_{i}^{k}\left\|e_{i}\left(t-\tau_{k}(t)\right)\right\|_{1}+a \sum_{i=1}^{N} \frac{\left\|e_{i}(t)\right\|_{1}^{p}}{n^{(p / 2)}}+b \sum_{i=1}^{N} \frac{\left\|e_{i}(t)\right\|_{1}^{q}}{n^{(q / 2)}}\right) .
\end{aligned}
$$

Substituting the calculating result (35) into (34), continue to calculate as follows:

$$
\begin{gathered}
\dot{V}(t) \leq \sum_{i=1}^{N}\left(\alpha+2\left|G_{i i}^{0}\right|\|D\|+\sum_{k^{\prime}=1}^{m}\left|G_{i i}^{k^{\prime}}\right|\left\|D_{\tau_{k^{\prime}}}\right\|-p_{i}\right) \\
\cdot\left\|e_{i}(t)\right\|_{1}+\sum_{i=1}^{N}\left(\beta-q_{i}\right)\left\|e_{i}(t-\tau(t))\right\|_{1} \\
+\sum_{i=1}^{N} \sum_{k=1}^{m}\left(3\left|G_{i i}^{k}\right|\left\|D_{\tau_{k}}\right\|-\xi_{i}^{k}\right)\left\|e_{i}\left(t-\tau_{k}(t)\right)\right\|_{1} \\
-a \sum_{i=1}^{N} \frac{\left\|e_{i}(t)\right\|_{1}^{p}}{n^{(p / 2)}}-b \sum_{i=1}^{N} \frac{\left\|e_{i}(t)\right\|_{1}^{q}}{n^{(q / 2)}} .
\end{gathered}
$$

Applying Lemma 4 and the error system to the process of the proof, we calculate the derivative of $V(t)$ step by step as follows:

$$
\begin{aligned}
\dot{V}(t) & =\sum_{i=1}^{N} \frac{\operatorname{sign}^{T}\left(e_{i}(t)\right) \dot{e}_{i}(t)}{\sqrt{n}} \\
\vdots & \sum_{i=1}^{N}\left(\alpha+2\left|G_{i i}^{0}\right|\|D\|+\sum_{k^{\prime}=1}^{m}\left|G_{i i}^{k^{\prime}}\right|\left\|D_{\tau_{k^{\prime}}}\right\|\right)\left\|e_{i}(t)\right\|_{1} \\
& +\sum_{i=1}^{N} \beta\left\|e_{i}(t-\tau(t))\right\|_{1} \\
& +\sum_{i=1}^{N} \sum_{k=1}^{m} 3\left|G_{i i}^{k}\right|\left\|D_{\tau_{k}} \mid\right\| e_{i}\left(t-\tau_{k}(t)\right) \|_{1} \\
& +\sum_{i=1}^{N} \frac{\operatorname{sign}^{T}\left(e_{i}(t)\right)}{\sqrt{n}} u_{i}(t) .
\end{aligned}
$$

The front part of the proof here is the same as that of Theorem 9. Hence, we omit the front part of proof and step to the calculating result (34) directly. The calculating result (34) contains many details. Firstly, in order to calculate conveniently later, we deal with the function $\sum_{i=1}^{\mathrm{N}}\left(\left(\operatorname{sign}^{T}\left(e_{i}(t)\right) / \sqrt{n}\right) u_{i}(t)\right)$ in (34). Then substitute the partial processing result into the previous result (34). We get 
Lemma 7, the fixed settling time can be calculated as follows:

$$
T_{0}=\frac{1}{a N^{1-p}(p-1)}+\frac{1}{b(1-q)} .
$$

The proof is completed here.

Remark 17. So far, lots of achievements for finite-time synchronization of complex networks have been obtained, while, to the best of our knowledge, there is little attention drawn to the fixed-time synchronization of the multilinked hybrid coupled complex networks with time-varying delays or few published papers in this field. That is why this study can draw our attention so much.

Corollary 18. Let all of Assumptions 1, 2, and 13 hold. Suppose that there exist positive constants $p_{i}, q_{i}$, and $\xi_{i}^{k}$ satisfying the following inequalities:

$$
\begin{aligned}
& p_{i} \geq \alpha+2 a_{0}\|D\|+\sum_{k^{\prime}=1}^{m} a_{k^{\prime}}\left\|D_{\tau_{k^{\prime}}}\right\|, \\
& q_{i} \geq \beta, \\
& \xi_{i}^{k} \geq 3 a_{k}\left\|D_{\tau_{k}}\right\|, \quad k=1,2, \ldots, m,
\end{aligned}
$$

where $p_{i}>0, q_{i}>0$, and $\xi_{i}^{k}>0$.

The controller designed to obtain the fixed-time synchronization is given as follows:

$$
\begin{aligned}
& u_{i}(t)=-\sqrt{n} \cdot \operatorname{sign}\left(\omega_{i}(t)\right)\left(p_{i}\left\|\omega_{i}(t)\right\|_{1}\right. \\
& +q_{i}\left\|\omega_{i}(t-\tau(t))\right\|_{1}+\sum_{k=1}^{m} \xi_{i}^{k}\left\|\omega_{i}\left(t-\tau_{k}(t)\right)\right\|_{1} \\
& \left.+\frac{a\left\|\omega_{i}(t)\right\|_{1}^{p}}{n^{(p / 2)}}+\frac{b\left\|\omega_{i}(t)\right\|_{1}^{q}}{n^{(q / 2)}}\right)
\end{aligned}
$$

where $a, b>0, p>1$, and $0<q<1$.

Then, the multilinked hybrid coupled complex networks (25) with control law (40) can realize fixed-time synchronization. The fixed settling time can be calculated by $T_{0}=$ $1 / a N^{1-p}(p-1)+1 / b(1-q)$.

Proof. The process of the proof for system (25) to obtain fixed-time synchronization is the same as that for Theorem 16. Hence, we omit it.

\section{Numerical Simulations}

We provide two simulation examples to illustrate the correctness and effectiveness of the results obtained by our paper in this section.
Example 1. This example considers the drive system (41) which is the 3-linked hybrid coupled complex networks and consists of 3 nodes. Each of the nodes is 2-dimensional.

$$
\begin{aligned}
& \dot{x}_{i}(t)= f\left(x_{i}(t)\right)+g\left(x_{i}(t-\tau(t))\right) \\
&+\sum_{j=1, j \neq i}^{3} G_{i j}^{0} D\left(x_{j}(t)-x_{i}(t)\right) \\
&+ \sum_{j=1, j \neq i}^{3} G_{i j}^{1} D_{\tau_{1}}\left(x_{j}\left(t-\tau_{1}(t)\right)-x_{i}(t)\right) \\
& \cdot \sum_{j=1, j \neq i}^{3} G_{i j}^{2} D_{\tau_{2}}\left(x_{j}\left(t-\tau_{2}(t)\right)-x_{i}(t)\right), \\
& i=1,2,3,
\end{aligned}
$$

where

$$
\begin{aligned}
f\left(x_{i}(t)\right) & \\
= & -\left[\begin{array}{ll}
1 & 0 \\
0 & 1
\end{array}\right]\left[\begin{array}{l}
x_{i 1}(t) \\
x_{i 2}(t)
\end{array}\right] \\
& +\left[\begin{array}{cc}
1+\frac{\pi}{8} & 1 \\
0.2 & 1+\frac{\pi}{8}
\end{array}\right]\left[\begin{array}{l}
f_{1}\left(x_{i 1}(t)\right) \\
f_{2}\left(x_{i 2}(t)\right)
\end{array}\right], \\
g & \left(x_{i}(t-\tau(t))\right) \\
= & {\left[\begin{array}{cc}
\sqrt{2} \frac{\pi}{8} 1.3 & 0.1 \\
0.1 & \sqrt{2} \frac{\pi}{8} 1.3
\end{array}\right]\left[\begin{array}{l}
f_{1}\left(x_{i 1}(t-\tau(t))\right) \\
f_{2}\left(x_{i 2}(t-\tau(t))\right)
\end{array}\right] . }
\end{aligned}
$$

And $f_{i}(x)=(|x+1|-|x-1|) / 2, i=1,2$. The time delays are chosen as follows:

$$
\begin{aligned}
\tau(t) & =\frac{e^{t}}{1+e^{t}}, \\
\tau_{1}(t) & =1.5(1-\cos (2 t)), \\
\tau_{2}(t) & =1+\sin (t) .
\end{aligned}
$$

The configuration matrices are chosen as follows:

$$
\begin{aligned}
& G_{0}=\left[\begin{array}{ccc}
-0.3 & 0.2 & 0.1 \\
0.2 & -0.6 & 0.4 \\
0.1 & 0.4 & -0.5
\end{array}\right], \\
& G_{1}=\left[\begin{array}{ccc}
-0.8 & 0.5 & 0.3 \\
0.5 & -0.7 & 0.2 \\
0.3 & 0.2 & -0.5
\end{array}\right], \\
& G_{2}=\left[\begin{array}{ccc}
-0.6 & 0.4 & 0.2 \\
0.4 & -0.9 & 0.5 \\
0.2 & 0.5 & -0.7
\end{array}\right] .
\end{aligned}
$$


The inner coupling matrices are chosen as follows:

$$
\begin{aligned}
D & =\left[\begin{array}{ll}
1 & 0 \\
2 & 1
\end{array}\right], \\
D_{\tau_{1}} & =\left[\begin{array}{cc}
3 & 1.2 \\
2 & 1
\end{array}\right], \\
D_{\tau_{2}} & =\left[\begin{array}{cc}
1.1 & 4 \\
1 & 2.5
\end{array}\right] .
\end{aligned}
$$

Correspondingly, the response system is given as follows:

$$
\begin{aligned}
\dot{y}_{i}(t)= & f\left(y_{i}(t)\right)+g\left(y_{i}(t-\tau(t))\right) \\
& +\sum_{j=1, j \neq i}^{3} G_{i j}^{0} D\left(y_{j}(t)-y_{i}(t)\right) \\
& +\sum_{j=1, j \neq i}^{3} G_{i j}^{1} D_{\tau_{1}}\left(y_{j}\left(t-\tau_{1}(t)\right)-y_{i}(t)\right) \\
& +\sum_{j=1, j \neq i}^{3} G_{i j}^{2} D_{\tau_{2}}\left(y_{j}\left(t-\tau_{2}(t)\right)-y_{i}(t)\right) \\
& +u_{i}(t), \quad i=1,2,3 .
\end{aligned}
$$

The initial conditions for simulation are given as follows:

$$
\begin{aligned}
& x_{1}(t)=\left(\begin{array}{c}
7-t \\
-1
\end{array}\right), \\
& x_{2}(t)=\left(\begin{array}{c}
3 \\
-5+t
\end{array}\right), \\
& x_{3}(t)=\left(\begin{array}{c}
2+2 t \\
-3
\end{array}\right), \\
& y_{1}(t)=\left(\begin{array}{c}
t+1 \\
-8
\end{array}\right), \\
& y_{2}(t)=\left(\begin{array}{c}
5 \\
2
\end{array}\right), \\
& y_{3}(t)=\left(\begin{array}{c}
-1 \\
-6
\end{array}\right),
\end{aligned}
$$

$$
t \in[-5,0] .
$$

We define the convergence errors as $\left\|e_{i}(t)\right\|_{1}=\left|y_{i 1}-x_{i 1}\right|+$ $\left|y_{i 2}-x_{i 2}\right|, i=1,2,3$.

We divide simulation Example 1 into three cases to illustrate our obtained results in more detail and in more normalized manner. Let $\alpha=3.9$ and $\beta=1$.

Case 1. We study the state trajectories of $x_{i j}(t)$ and $y_{i j}(t)(i=$ $1,2,3 ; j=1,2)$ when the drive-response systems are without control inputs, which are showed in Figure 1. Moreover, the

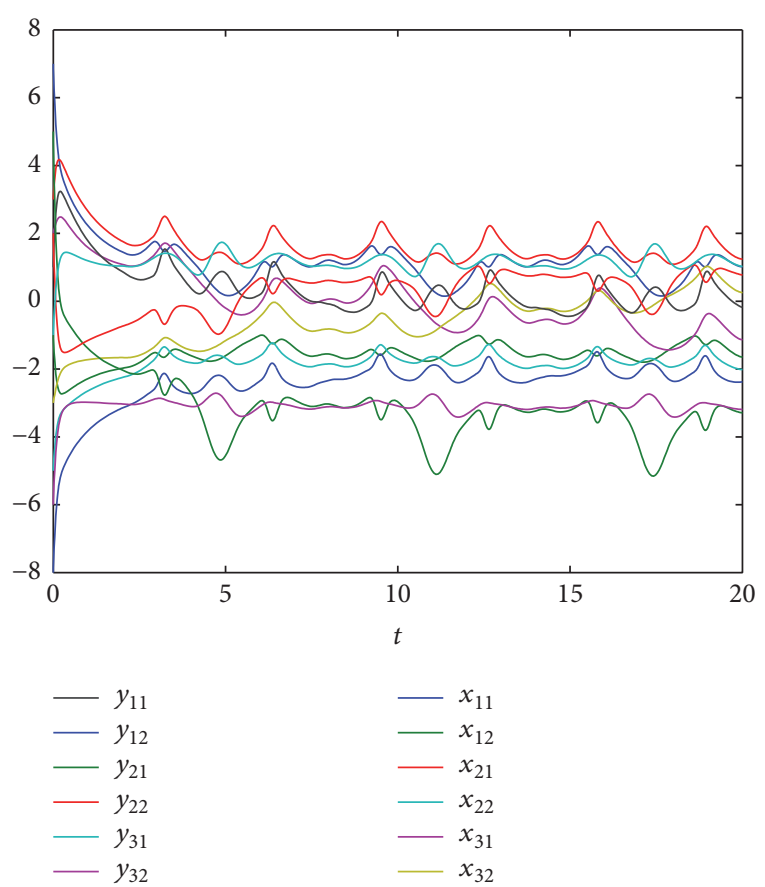

Figure 1: The state trajectories of system (41) and system (46) without control inputs.
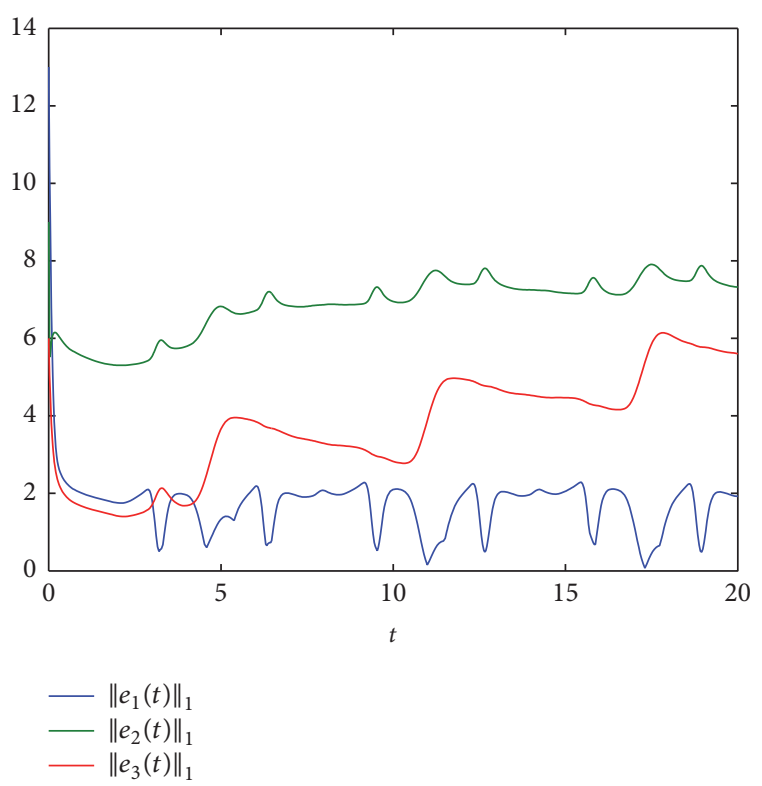

FIgURE 2: The convergence errors between system (41) and system (46) without control inputs.

trajectories of $\left\|e_{i}(t)\right\|_{1}, i=1,2,3$ without control inputs can be seen in Figure 2.

Case 2. We simulate the result of Theorem 9 when inequalities in (18) are satisfied. We choose the values of the parameters as follows: $p_{1}=12, q_{1}=1, \xi_{1}^{1}=10$, and $\xi_{1}^{2}=9$; $p_{2}=14, q_{2}=1, \xi_{2}^{1}=9$, and $\xi_{2}^{2}=14 ; p_{3}=12, q_{3}=1$, $\xi_{3}^{1}=6$, and $\xi_{3}^{2}=11 ; k_{0}=2 ; \eta=1 / 2$. Let $t_{0}=0$. According to 


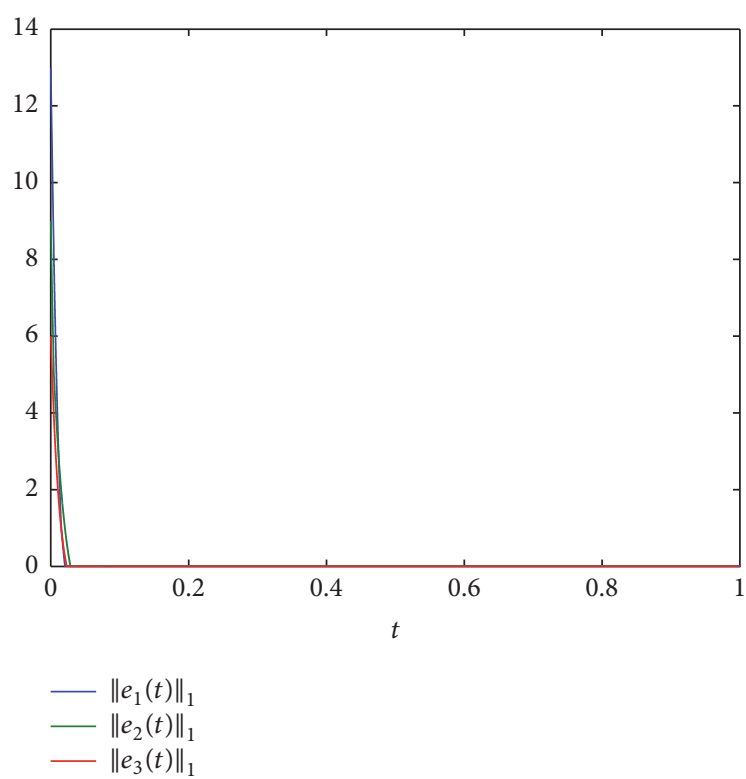

Figure 3: The convergence errors between system (41) and system (46) under controller (17).

Theorem 9, we can calculate that the value of the settling time $t_{1}$ is 4.4496 . The simulation of finite-time synchronization for the drive-response systems (41) and (46) in settling time $t_{1}$ with control inputs (17) is showed in Figure 3.

Case 3. We simulate the result of Theorem 16 when inequalities in (32) are satisfied. We choose the values of the parameters as follows: $p_{1}=12, q_{1}=1, \xi_{1}^{1}=10$, and $\xi_{1}^{2}=9$; $p_{2}=14, q_{2}=1, \xi_{2}^{1}=9$, and $\xi_{2}^{2}=14 ; p_{3}=12, q_{3}=1$, $\xi_{3}^{1}=6$, and $\xi_{3}^{2}=11 ; a=1, b=1, p=2$, and $q=1 / 2$. According to Theorem 16, we can calculate that the value of the fixed settling time $T_{0}$ is 5 . The simulation of fixed-time synchronization for the drive-response systems (41) and (46) in fixed settling time $T_{0}$ with control inputs (31) is showed in Figure 4.

Example 2. This example considers the drive system (48) which is the 3-linked hybrid coupled complex network and consists of 3 nodes. Each of the nodes is 2-dimensional. The system which is similar to (41) is given as follows:

$$
\begin{aligned}
\dot{x}_{i}(t)= & f\left(x_{i}(t)\right)+g\left(x_{i}(t-\tau(t))\right) \\
& +\sum_{j=1, j \neq i}^{3} G_{i j}^{0} D\left(x_{j}(t)-x_{i}(t)\right) \\
& +\sum_{j=1, j \neq i}^{3} G_{i j}^{1} D_{\tau_{1}}\left(x_{j}\left(t-\tau_{1}(t)\right)-x_{i}(t)\right) \\
& \cdot \sum_{j=1, j \neq i}^{3} G_{i j}^{2} D_{\tau_{2}}\left(x_{j}\left(t-\tau_{2}(t)\right)-x_{i}(t)\right) \\
& +u_{i}(t), \quad i=1,2,3,
\end{aligned}
$$

where $u_{i}(t)$ denotes suitable control inputs.

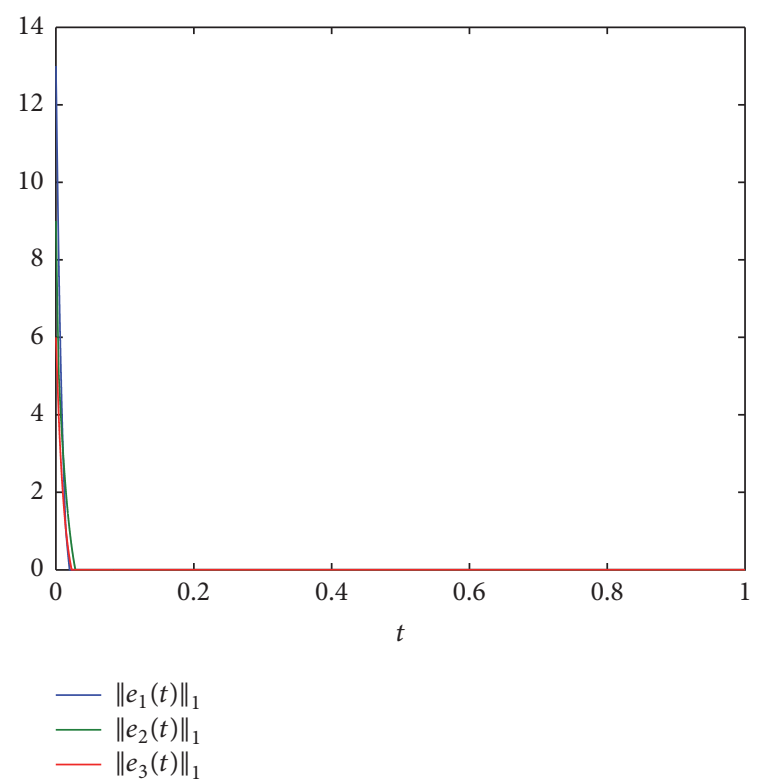

FIGURE 4: The convergence errors between system (41) and system (46) under controller (31).

And we will use the same $f(\cdot), g(\cdot), f_{i}(x), D, D_{\tau_{1}}, D_{\tau_{2}}$, $\tau(t), \tau_{1}(t)$, and $\tau_{2}(t)$ given in Example 1.

The configuration matrices are chosen as follows:

$$
\begin{aligned}
& G_{0}=\left[\begin{array}{ccc}
-0.3 & 0.15 & 0.15 \\
0.15 & -0.3 & 0.15 \\
0.15 & 0.15 & -0.3
\end{array}\right], \\
& G_{1}=\left[\begin{array}{ccc}
-0.4 & 0.2 & 0.2 \\
0.2 & -0.4 & 0.2 \\
0.2 & 0.2 & -0.4
\end{array}\right], \\
& G_{2}=\left[\begin{array}{lll}
-0.5 & 0.25 & 0.25 \\
0.25 & -0.5 & 0.25 \\
0.25 & 0.25 & -0.5
\end{array}\right] .
\end{aligned}
$$

According to (26), the synchronized state model is given as follows:

$$
\begin{aligned}
\dot{s}(t)= & f(s(t))+g(s(t-\tau(t))) \\
& -(-0.4) D_{\tau_{1}}\left(s\left(t-\tau_{1}(t)\right)-s(t)\right) \\
& -(-0.5) D_{\tau_{2}}\left(s\left(t-\tau_{2}(t)\right)-s(t)\right) .
\end{aligned}
$$

The initial conditions for simulation are given as follows:

$$
\begin{aligned}
s(t) & =\left(\begin{array}{c}
8-t \\
-1
\end{array}\right), \\
x_{1}(t) & =\left(\begin{array}{c}
3 \\
-5+t
\end{array}\right),
\end{aligned}
$$




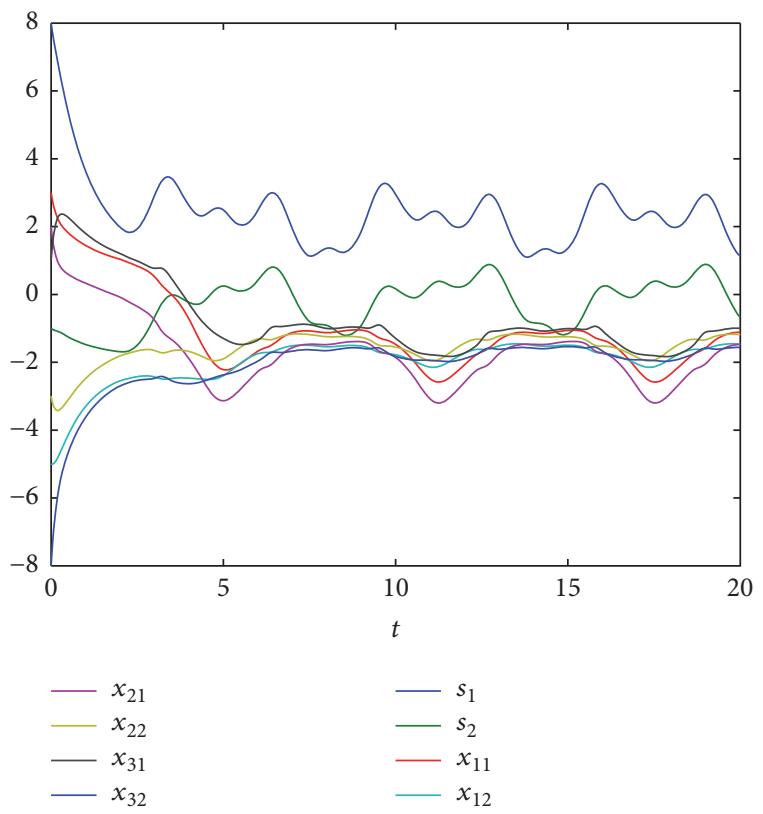

FIGURE 5: The state trajectories of systems (48) and (50) without control inputs.

$$
\begin{aligned}
& x_{2}(t)=\left(\begin{array}{c}
2+2 t \\
-3
\end{array}\right), \\
& x_{3}(t)=\left(\begin{array}{c}
t+1 \\
-8
\end{array}\right), \\
& t \in[-5,0] .
\end{aligned}
$$

In Example 2, we define the convergence errors as $\left\|\omega_{i}(t)\right\|_{1}=\left|x_{i 1}-s_{1}\right|+\left|x_{i 2}-s_{2}\right|, i=1,2,3$. And we divide simulation Example 2 into three cases to illustrate our obtained results in more detail and in more normalized manner.

Case 1 . We study the state trajectories of $x_{i j}(t)$ and $s_{j}(t)(i=$ $1,2,3 ; j=1,2)$ when systems (48) and (50) are without control inputs, which are showed in Figure 5. Moreover, the trajectories of $\left\|\omega_{i}(t)\right\|_{1}, \quad i=1,2,3$, without control inputs can be seen in Figure 6.

Case 2. We simulate the result of Corollary 14 . We choose the values of the parameters as follows: $p_{1}=10, q_{1}=1$, $\xi_{1}^{1}=5$, and $\xi_{1}^{2}=8 ; p_{2}=10$ and $q_{2}=1, \xi_{2}^{1}=5$, and $\xi_{2}^{2}=8 ; p_{3}=10, q_{3}=1, \xi_{3}^{1}=5$, and $\xi_{3}^{2}=8 ; k_{0}=2$ and $\eta=1 / 2$. The simulation of finite-time synchronization for systems (48) and (50) in settling time $t_{1}$ (through calculating, $\left.t_{1}=4.6819\right)$ with control inputs (29) is showed in Figure 7.

Case 3. We simulate the result of Corollary 18 when inequalities in (39) are satisfied. We choose the values of the parameters as follows: $p_{1}=10, q_{1}=1, \xi_{1}^{1}=5$, and $\xi_{1}^{2}=8$; $p_{2}=10, q_{2}=1, \xi_{2}^{1}=5$, and $\xi_{2}^{2}=8 ; p_{3}=10, q_{3}=1$,

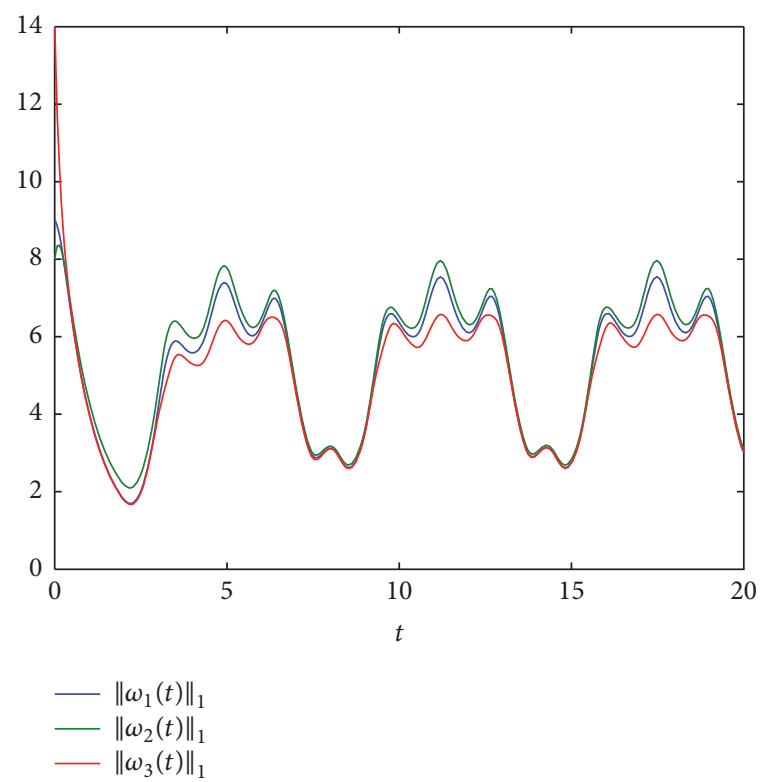

FIgURE 6: The convergence errors between system (48) and system (50) without control inputs.

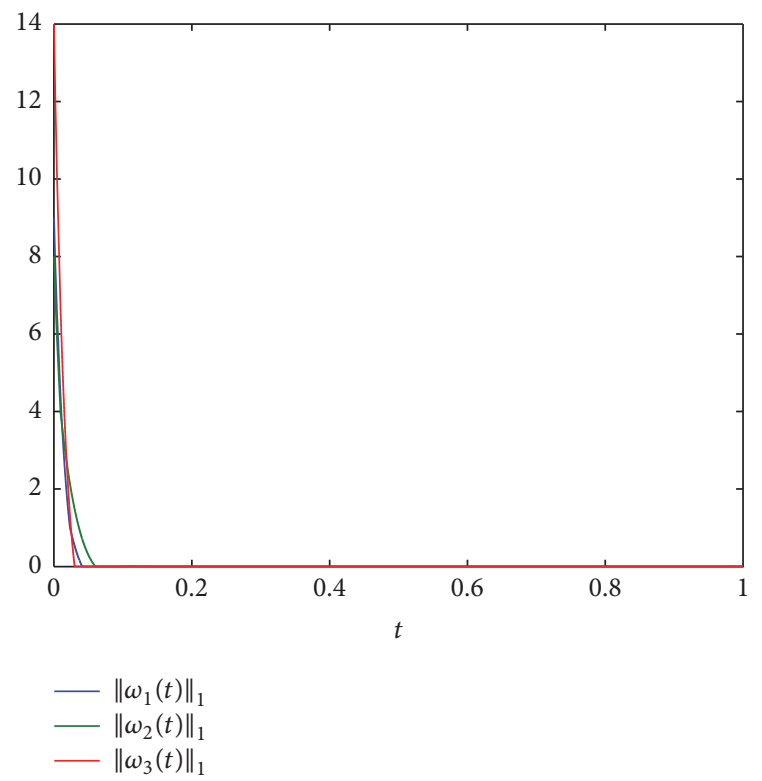

FIGURE 7: The convergence errors of systems (48) and (50) under controller (29).

$\xi_{3}^{1}=5$, and $\xi_{3}^{2}=8 ; a=1, b=1, p=2$, and $q=1 / 2$. According to Corollary 18, we can calculate that the value of the fixed settling time $T_{0}$ is 5 . The simulation of fixed-time synchronization for the drive-response systems (48) and (50) in fixed settling time $T_{0}$ with control inputs (40) is showed in Figure 8.

\section{Conclusions}

In this paper, we study the finite-time synchronization of new multilinked hybrid coupled complex dynamical networks 


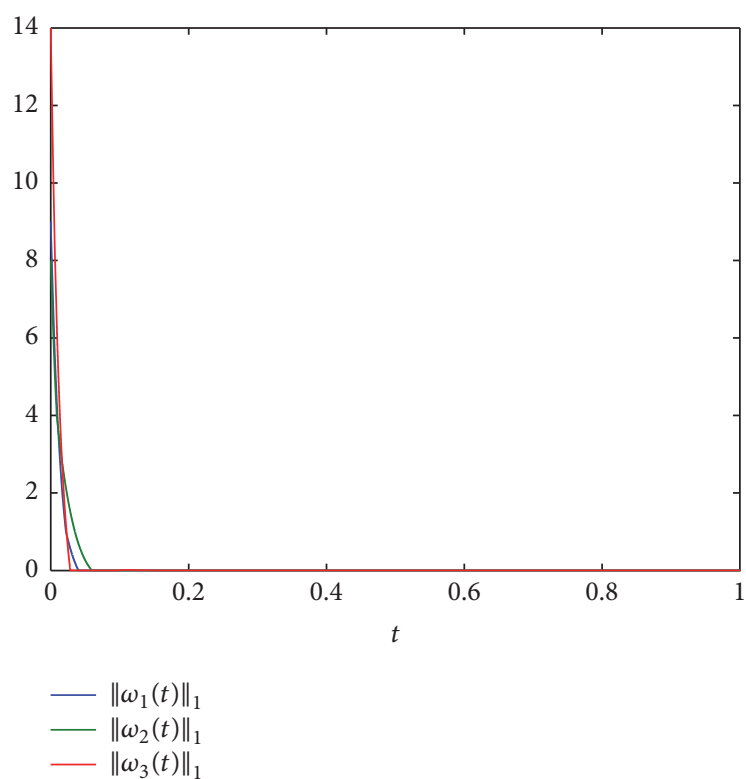

Figure 8: The convergence errors between systems (48) and (50) under controller (40).

with one single time-varying delay coupling for the first time. Furthermore, we study the fixed-time synchronization of new multilinked hybrid coupled complex dynamical networks with one single time-varying delay coupling for the first time. Two suitable feedback controllers are designed in order to obtain the finite/fixed-time synchronization. Several synchronization criteria are proposed through strict calculating and theorem derivation. Finally, we use two appropriate numerical examples to illustrate the correctness and effectiveness of the results proposed in this paper. The problem of fixed-time synchronization and finite-time synchronization for the hybrid coupled dynamical networks whose coupling terms contain only one transmittal time-varying delay comes naturally for multilinked systems which simulate the real world in closer and more realistic way. It is the first time to study this exact problem and the significance of solving the problem that make our work interesting and meaningful.

\section{Competing Interests}

The authors declare that they have no competing interests.

\section{Acknowledgments}

The work is supported by the National Key Research and Development Program (Grant no. 2016YFB0800602) and the National Natural Science Foundation of China (Grant nos. 61472045 and 61573067).

\section{References}

[1] H. Qin, J. Ma, W. Jin, and C. Wang, "Dynamics of electric activities in neuron and neurons of network induced by autapses," Science China Technological Sciences, vol. 57, no. 5, pp. 936-946, 2014.
[2] X. F. Wang, "Complex networks: topology, dynamics and synchronization," International Journal of Bifurcation and Chaos, vol. 12, no. 5, pp. 885-916, 2002.

[3] T. Li and B. Rao, "Asymptotic controllability and asymptotic synchronization for a coupled system of wave equations with Dirichlet boundary controls," Asymptotic Analysis, vol. 86, no. 3-4, pp. 199-226, 2014.

[4] S. Wen, S. Chen, and C. Wang, "Global synchronization in complex networks consisted of systems with the property of $\mathrm{x}_{\mathrm{k}}$ leading asymptotic stability," Physics Letters A, vol. 372, no. 17, pp. 3021-3026, 2008.

[5] S. K. Agrawal and S. Das, "Projective synchronization between different fractional-order hyperchaotic systems with uncertain parameters using proposed modified adaptive projective synchronization technique," Mathematical Methods in the Applied Sciences, vol. 37, no. 14, pp. 2164-2176, 2014.

[6] W. Wang, H. Peng, L. Li, J. Xiao, and Y. Yang, "Finite-time function projective synchronization in complex multi-links networks with time-varying delay," Neural Processing Letters, vol. 41, no. 1, pp. 71-88, 2015.

[7] Z. Wu, J. Duan, and X. Fu, "Complex projective synchronization in coupled chaotic complex dynamical systems," Nonlinear Dynamics, vol. 69, no. 3, pp. 771-779, 2012.

[8] L. J. Banu and P. Balasubramaniam, "Synchronisation of discrete-time complex networks with randomly occurring uncertainties, nonlinearities and time-delays," International Journal of Systems Science, vol. 45, no. 7, pp. 1427-1450, 2014.

[9] B. Li, "Finite-time synchronization for complex dynamical networks with hybrid coupling and time-varying delay," Nonlinear Dynamics, vol. 76, no. 2, pp. 1603-1610, 2014.

[10] O. Naifar, A. Ben Makhlouf, M. Hammami, and A. Ouali, "State feedback control law for a class of nonlinear timevarying system under unknown time-varying delay," Nonlinear Dynamics, vol. 82, no. 1-2, pp. 349-355, 2015.

[11] W. Cui, J.-A. Fang, W. Zhang, and X. Wang, "Finite-time cluster synchronisation of Markovian switching complex networks with stochastic perturbations," IET Control Theory \& Applications, vol. 8, no. 1, pp. 30-41, 2014.

[12] L. Li, Z. Tu, J. Mei, and J. Jian, "Finite-time synchronization of complex delayed networks via intermittent control with multiple switched periods," Nonlinear Dynamics, vol. 85, no. 1, pp. 375-388, 2016.

[13] J. Sun, Y. Shen, X. Wang, and J. Chen, "Finite-time combinationcombination synchronization of four different chaotic systems with unknown parameters via sliding mode control," Nonlinear Dynamics, vol. 76, no. 1, pp. 383-397, 2014.

[14] Y. Chen, M. Li, and Z. Cheng, "Global anti-synchronization of master-slave chaotic modified Chua's circuits coupled by linear feedback control," Mathematical \& Computer Modelling, vol. 52, no. 3-4, pp. 567-573, 2010.

[15] Q. Lin and X. Wu, "The sufficient criteria for global synchronization of chaotic power systems under linear state-error feedback control," Nonlinear Analysis: Real World Applications, vol. 12, no. 3, pp. 1500-1509, 2011.

[16] B. Wang, J. Xue, F. Wu, and D. Zhu, "Stabilization conditions for fuzzy control of uncertain fractional order non-linear systems with random disturbances," IET Control Theory \& Applications, vol. 10, no. 6, pp. 637-647, 2016.

[17] K. Zhang and M. A. Demetriou, "Adaptation and optimization of the synchronization gains in the adaptive spacecraft attitude synchronization," Aerospace Science \& Technology, vol. 46, pp. 116-123, 2015. 
[18] Y.-W. Wang, J.-W. Xiao, C. Wen, and Z.-H. Guan, "Synchronization of continuous dynamical networks with discrete-time communications," IEEE Transactions on Neural Networks, vol. 22, no. 12, pp. 1979-1986, 2011.

[19] L. Wang, Y. Shen, and G. Zhang, "General decay synchronization stability for a class of delayed chaotic neural networks with discontinuous activations," Neurocomputing, vol. 179, pp. 169175, 2016.

[20] J. Shen and J. Cao, "Finite-time synchronization of coupled neural networks via discontinuous controllers," Cognitive Neurodynamics, vol. 5, no. 4, pp. 373-385, 2011.

[21] Y. Zhang and Z. He, "A secure communication scheme based on cellular neural networks," in Proceedings of the IEEE International Conference on Intelligent Processing Systems (ICIPS '97), vol. 1, pp. 521-524, Beijing, China, October 1997.

[22] A. Polyakov, "Nonlinear feedback design for fixed-time stabilization of linear control systems," IEEE Transactions on Automatic Control, vol. 57, no. 8, pp. 2106-2110, 2012.

[23] C. W. Wu, "Synchronization in arrays of coupled nonlinear systems with delay and nonreciprocal time-varying coupling," IEEE Transactions on Circuits and Systems II: Express Briefs, vol. 52, no. 5, pp. 282-286, 2005.

[24] J. Mei, M. Jiang, W. Xu, and B. Wang, "Finite-time synchronization control of complex dynamical networks with time delay," Communications in Nonlinear Science \& Numerical Simulation, vol. 18, no. 9, pp. 2462-2478, 2013.

[25] J. Huang, C. Li, T. Huang, and X. He, "Finite-time lag synchronization of delayed neural networks," Neurocomputing, vol. 139, pp. 145-149, 2014.

[26] W. He and J. Cao, "Global synchronization in arrays of coupled networks with one single time-varying delay coupling," Physics Letters A, vol. 373, no. 31, pp. 2682-2694, 2009.

[27] C. W. Wu, Synchronization in Complex Networks of Nonlinear Dynamical Systems, World Scientific, Singapore, 2007.

[28] A. L. Barabsi, Linked: The New Science of Networks, Persus Publishing, Boston, Mass, USA, 2002.

[29] J. Scott, Social Network Analysis, Sage, 2000.

[30] W. Wang, L. Li, H. Peng, J. Xiao, and Y. Yang, "Stochastic synchronization of complex network via a novel adaptive nonlinear controller," Nonlinear Dynamics, vol. 76, no. 1, pp. 591-598, 2014.

[31] J.-W. Yi, Y.-W. Wang, J.-W. Xiao, and Y. Huang, "Synchronisation of complex dynamical networks with additive stochastic timevarying delays," International Journal of Systems Science, vol. 47, no. 5, pp. 1221-1229, 2016.

[32] T. Wang, Y. Ding, L. Zhang, and K. Hao, "Adaptive feedback synchronisation of complex dynamical network with discretetime communications and delayed nodes," International Journal of Systems Science, vol. 47, no. 11, pp. 2563-2571, 2016.

[33] Q. Zhang, J. Lu, J. Lu, and C. K. Tse, "Adaptive feedback synchronization of a general complex dynamical network with delayed nodes," IEEE Transactions on Circuits and Systems II: Express Briefs, vol. 55, no. 2, pp. 183-187, 2008.

[34] H. Peng, N. Wei, L. Li, W. Xie, and Y. Yang, "Models and synchronization of time-delayed complex dynamical networks with multi-links based on adaptive control," Physics Letters, Section A, vol. 374, no. 23, pp. 2335-2339, 2010.

[35] M. Zheng, L. Li, H. Peng et al., "Finite-time synchronization of complex dynamical networks with multi-links via intermittent controls," The European Physical Journal B, vol. 89, article 43, 12 pages, 2016.
[36] H. Zhao, L. Li, H. Peng, J. Xiao, Y. Yang, and M. Zheng, "Impulsive control for synchronization and parameters identification of uncertain multi-links complex network," Nonlinear Dynamics, vol. 83, no. 3, pp. 1437-1451, 2016.

[37] W. Yu, G. Chen, and J. Cao, "Adaptive synchronization of uncertain coupled stochastic complex networks," Asian Journal of Control, vol. 13, no. 3, pp. 418-429, 2011.

[38] H. K. Khalil and J. W. Grizzle, Nonlinear Systems, Prentice Hall, Upper Saddle River, NJ, USA, 2002.

[39] F. H. Clarke, "Nonsmooth analysis and optimization," in Proceedings of the International Congress of Mathematicians, vol. 5, pp. 847-853, Warszawa, Poland, August 1983.

[40] Y. Tang, "Terminal sliding mode control for rigid robots," Automatica, vol. 34, no. 1, pp. 51-56, 1998.

[41] Y. Shen, Y. Huang, and J. Gu, "Global finite-time observers for Lipschitz nonlinear systems," Institute of Electrical and Electronics Engineers. Transactions on Automatic Control, vol. 56, no. 2, pp. 418-424, 2011. 


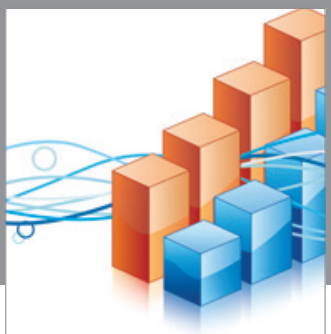

Advances in

Operations Research

vatem alat4

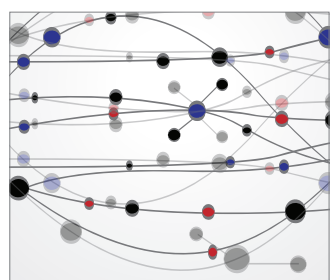

\section{The Scientific} World Journal
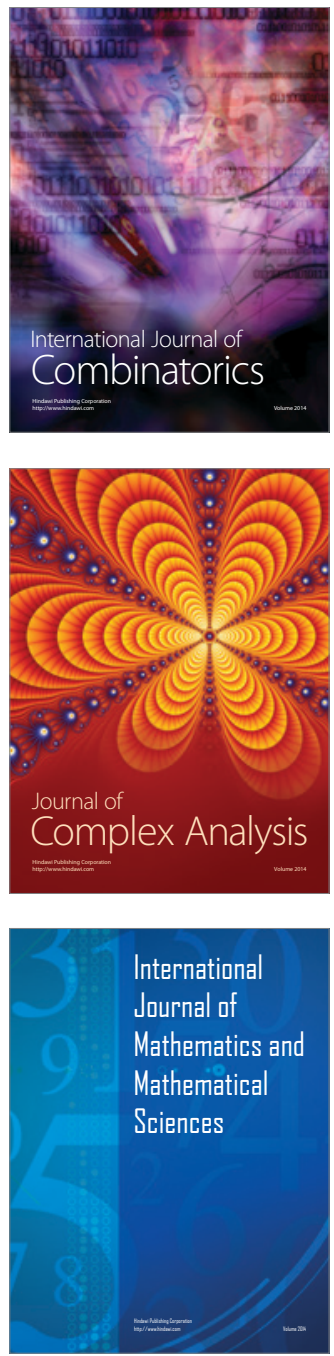
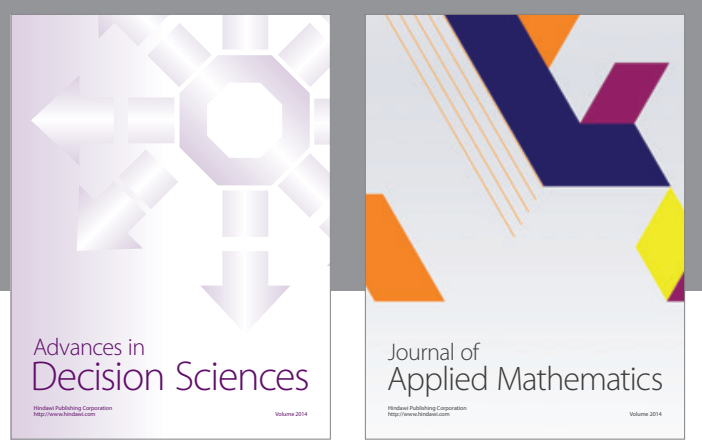

Algebra

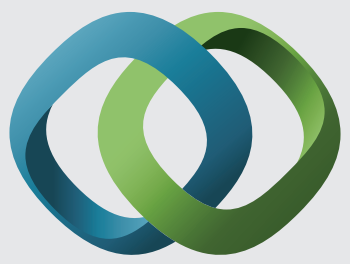

\section{Hindawi}

Submit your manuscripts at

https://www.hindawi.com
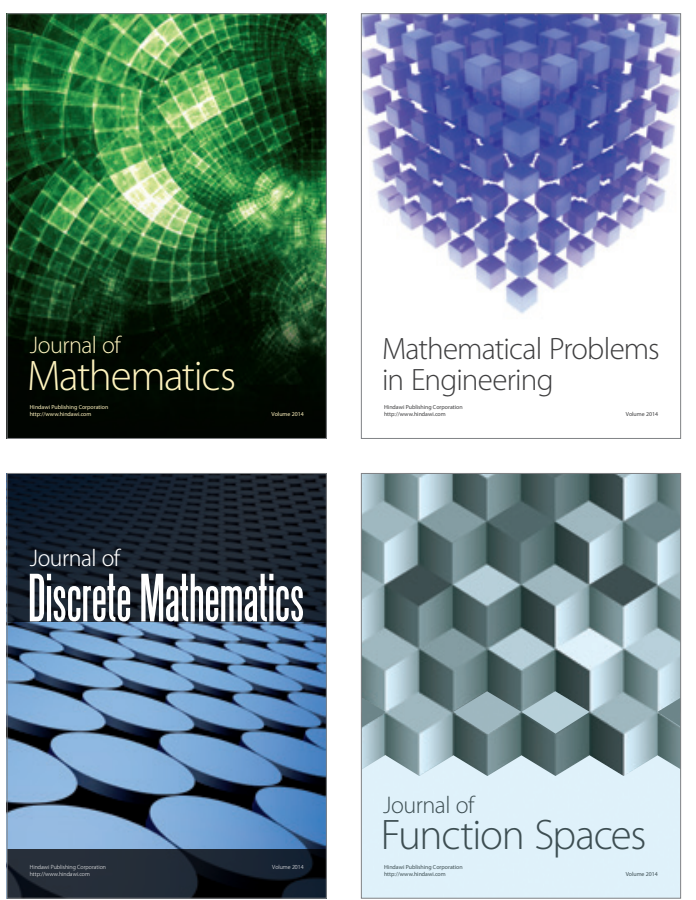

Mathematical Problems in Engineering
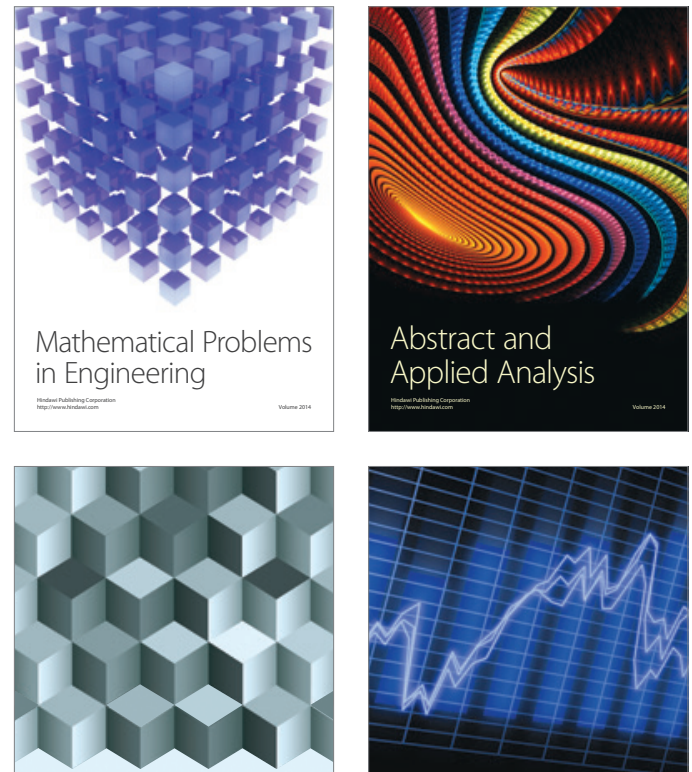

Journal of

Function Spaces

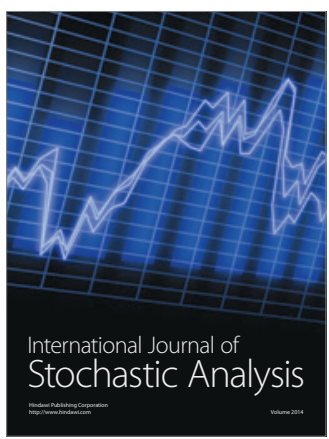

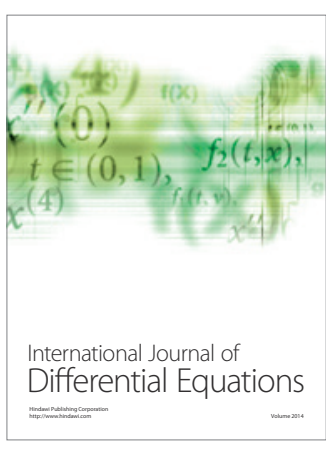
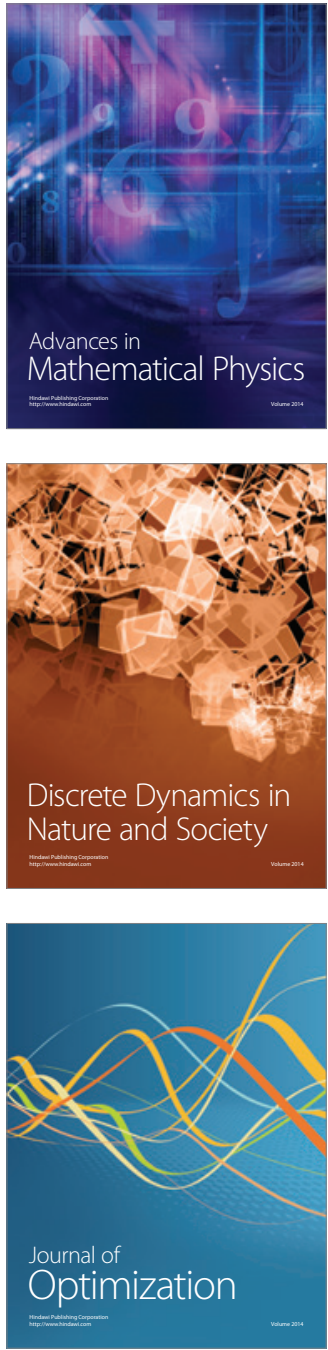